\title{
Parámetros Productivos de Cuyes Criados con Dietas Suplementadas con Aceite de Pescado y Semillas de Sacha Inchi
}

\author{
Productive Parameters of Guinea Pigs Raised with Diets Supplemented \\ with Fish OIL ANd SACha Inchi Seeds
}

\author{
Jorge Guevara V. ${ }^{1,4}$, Sergio Rojas M. $^{2}$, Fernando Carcelén C. ${ }^{3}$, \\ Sandra Bezada $\mathbf{Q}^{3}$, Teresa Arbaiza . $^{3}$
}

\section{Resumen}

El objetivo del estudio fue determinar los parámetros productivos de cuyes con el uso de dietas suplementadas con aceite de pescado y semilla de sacha inchi. Se utilizaron 48 cuyes machos de 42 días de edad, con un peso inicial de $615 \mathrm{~g}$. Los cuyes fueron asignados al azar a 4 tratamientos con 3 repeticiones (pozas) de 4 cuyes cada una. Los tratamientos dietéticos fueron: T0: Control, T1: Dieta suplementada con 1.0\% de aceite de pescado; T2: Dieta suplementada con 4.0\% de semilla de sacha inchi; y T3: Dieta suplementada con $1.0 \%$ de aceite de pescado $+4.0 \%$ de semilla de sacha inchi. La fase experimental tuvo una duración de 28 días. Se evaluó la ganancia de peso, consumo de alimento, conversión alimenticia y rendimiento de carcasa. El promedio de ganancia de peso individual en las 4 semanas varió entre 315 y 353 g por tratamiento, el consumo varió entre 1172 y $1224 \mathrm{~g}$ de materia seca, la conversión alimenticia varió entre 3.53 y 3.73 y el rendimiento de carcasa varió entre 69.4 y $71.7 \%$, sin diferencia estadística entre tratamientos.

Palabras clave: cuy, parámetros productivos, aceite de pescado, sacha inchi

1 Escuela Académica Profesional de Ingeniería Agroindustrial, Universidad Nacional Mayor de San Marcos, Lima, Perú

2 Departamento de Nutrición, Facultad de Zootecnia, Universidad Nacional Agraria La Molina, Lima, Perú

3 Laboratorio de Bioquímica, Nutrición y Alimentación Animal, Facultad de Medicina Veterinaria, Universidad Nacional Mayor de San Marcos, Lima, Perú

4 E-mail: guevaravet@hotmail.com

Recibido: 1 de octubre de 2015

Aceptado para publicación: 19 de julio de 2016 
The aim of the study was to determine the productive parameters of guinea pigs raised with diets supplemented with fish oil and sacha inchi seeds. Forty eight male guinea pigs of 42 days old were used, with an initial body weight of $615 \mathrm{~g}$. The guinea pigs were randomly assigned to 4 treatments with 3 repetitions (rearing pools) of 4 animals each. Dietary treatments were: T0: Control, T1: diet supplemented with $1.0 \%$ fish oil; T2: diet supplemented with $4.0 \%$ sacha inchi seed; and T3: diet supplemented with $1.0 \%$ fish oil $+4.0 \%$ sacha inchi seed. The experimental phase lasted 28 days. Body weight gain, feed intake, feed conversion and carcass yield were evaluated. The average of individual body weight gain ranged between 315 and $353 \mathrm{~g}$ per treatment, consumption ranged between 1172 and $1224 \mathrm{~g}$ dry matter, feed conversion ranged between 3.53 and 3.73 and carcass yield varied between 69.4 and $71.7 \%$ without statistical differences between treatments.

Key words: guinea pig, production parameters, fish oil, sacha inchi seed

\section{INTRODUCCIÓN}

El cuy (Cavia porcellus) es un roedor originario de los Andes, utilizado como alimento en una extensa región comprendida por Bolivia, Perú, Ecuador y Colombia. Su crianza ha suscitado un gran interés en el Perú, no solo por ser una valiosa fuente de nutrientes, sino también como una fuente de ingreso económico (Camino e Hidalgo, 2014). Asimismo, es considerado por la ONU y la FAO como una fuente de seguridad alimenticia para la población de escasos recursos económicos (Sánchez et al., 2009).

Estudios realizados con sacha inchi (Plukenetia volubilis) muestran que esta planta es una fuente importante de aceite y proteína (Gorriti y Arroyo, 2010), quizá comparable a otras de alto reconocimiento como la soya (Glycine max), el maíz (Zea mays), el maní (Arachis hypogaea), el girasol (Helianthus annuus) y la palma (Arecaceae). Otras investigaciones muestran al aceite generado por la planta con posibilidades de competir con el aceite de oliva, el de mayor demanda comercial a nivel mundial (Follegatti et al., 2009), dado su alto contenido de ácidos grasos omega (FAco)
(Gutiérrez et al., 2011). A esto, se le puede agregar la posibilidad de encontrar ácidos grasos funcionales, que además de complementar la aptitud nutricional de los ácidos grasos poliinsaturados (PUFA), actúan benéficamente sobre algunas actividades del organismo humano (Hosamani y Ganjihal, 2003).

Al no existir a la fecha información sobre trabajos de investigación empleando sacha inchi en la dieta de cuyes, se planteó la siguiente investigación, cuyo objetivo fue determinar los parámetros productivos de cuyes con el uso de dietas suplementadas con aceite de pescado y semilla de sacha inchi.

\section{Materiales y Métodos}

El presente trabajo se llevó a cabo en la Sala Experimental de Cuyes del Programa de Investigación en Carnes de la Facultad de Zootecnia, Universidad Nacional Agraria La Molina (UNALM), Lima.

Se emplearon 48 cuyes machos de raza Perú, con una edad promedio de 42 días y peso inicial de $615 \mathrm{~g}$. Los cuyes provinieron 
de la granja de cuyes de Cieneguilla, UNALM. Fueron pesados individualmente y distribuidos al azar, formando grupos homogéneos de cuatro animales por poza. Se utilizó un diseño completamente al azar con cuatro tratamientos y tres repeticiones cada uno, donde una repetición fue representada por un grupo de cuatro cuyes alojados en una poza.

Las pozas $(\mathrm{n}=12)$ eran de material noble y revestidas con cemento, de $1.0 \mathrm{~m}$ por lado y $0.37 \mathrm{~m}$ de altura. En los pisos de las pozas se colocó una cama de coronta molida de $5 \mathrm{~cm}$ de espesor para un mejor control de la humedad.

Para el suministro de concentrado, en las primeras dos semanas se utilizaron comederos de arcilla enlozada de forma rectangular, con capacidad de $250 \mathrm{~g}$; luego fueron cambiadas por tolvas plásticas pequeñas de $3 \mathrm{~kg}$ de capacidad hasta el final de la fase experimental. Como bebederos se utilizaron pocillos de porcelana con capacidad de $200 \mathrm{ml}$ de agua por poza. Para la medición del peso vivo de los animales, alimento balanceado, forraje y carcasa se utilizó una balanza de $5 \mathrm{~kg}$ de capacidad con $2 \mathrm{~g}$ de aproximación.

Todos los cuyes fueron alimentados con concentrado y rastrojo de brócoli (Brassica oleracea var. itálica) como forraje verde. Este último fue suministrado a razón de $10 \%$ del peso vivo y ofrecido en la poza de los animales. El forraje fue consumido en su totalidad sin dejar residuos. Se mantuvo un registro semanal de la cantidad de forraje utilizado.

En las primeras cuatro semanas consumieron una ración de crecimiento $(2730 \mathrm{Kcal}$ ED/kg y $18 \%$ de proteína cruda), mientras que en las últimas cuatro semanas recibieron una de las cuatro dietas experimentales (etapa de acabado): T0: Dieta Control (sin aceite de pescado ni semilla de sacha inchi); T1: Dieta suplementada con $1.0 \%$ de aceite de pescado; T2: Dieta suplementada con $4.0 \%$ de semilla de sacha inchi; y T3: Dieta suplementada con $1.0 \%$ de aceite de pescado y $4.0 \%$ de semilla de sacha inchi. Las dietas experimentales en los cuatro tratamientos contenían $2760 \mathrm{Kcal}$ de ED/kg y 16\% de proteína cruda.

Las dietas, en forma de harina, fueron preparadas cada cuatro días y se ofrecieron a los animales diariamente ad libitum entre las 09:30 y las 10:30 horas. Las dietas fueron colocadas en bolsas plásticas convenientemente identificadas por tratamiento y almacenadas en refrigeración. Se suministró agua fresca y limpia a todos los animales diariamente entre las 10:00 y las 14:00 horas; luego se retiraban los bebederos hasta el siguiente día para evitar exceso de humedad en las camas.

\section{Los parámetros evaluados fueron:}

- Ganancia de peso. Se midió el peso vivo de los animales al inicio del experimento y luego semanalmente. El peso se midió a la misma hora y con el animal en ayunas. La ganancia de peso se determinó por el peso vivo ganado en cada semana.

- Consumo de alimento. El consumo se estimó en las mismas fechas del registro del peso. El consumo se midió por poza considerando el alimento ofrecido menos el residuo (alimento sobrante en el comedero). El consumo fue expresado como consumo diario en gramos de materia seca.

- Conversión alimenticia. Se determinó a través del consumo semanal dividido entre la ganancia de peso semanal.

- Rendimiento de carcasa. Se determinó en 4 cuyes por tratamiento (total: 16 cuyes) elegidos al azar. Los animales estuvieron en ayuno de 12 horas antes del sacrificio. La carcasa incluyó piel, cabeza, miembros anteriores y posteriores y vísceras rojas (corazón, pulmones, hígado y riñones). El rendimiento de la carcasa se calculó mediante el peso vivo menos el peso de la carcasa y se expresó en porcentaje. 
Cuadro 1. Ganancias de peso semanal (g) por cuy con dietas suplementadas con aceite de pescado y sacha inchi (Plukenetia volubilis)

\begin{tabular}{|c|c|c|c|c|c|c|c|}
\hline \multirow{2}{*}{ Tratamiento ${ }^{1}$} & \multirow{2}{*}{$\begin{array}{l}\text { Peso } \\
\text { inicial }\end{array}$} & \multicolumn{4}{|c|}{ Semanas de suplemento } & \multirow{2}{*}{$\begin{array}{l}\text { Peso } \\
\text { final }\end{array}$} & \multirow{2}{*}{$\begin{array}{c}\text { Ganancia } \\
\text { de peso }\end{array}$} \\
\hline & & 1 & 2 & 3 & 4 & & \\
\hline T0 & 597.7 & 57.3 & 94.7 & 81.7 & 90.3 & 921.7 & $324.0^{\mathrm{a}}$ \\
\hline $\mathrm{T} 1$ & 612.3 & 66.7 & 98.7 & 87.0 & 90.3 & 955.0 & $342.7^{\mathrm{a}}$ \\
\hline $\mathrm{T} 2$ & 629.0 & 70.7 & 94.3 & 90.7 & 98.3 & 983.0 & $353.0^{\mathrm{a}}$ \\
\hline $\mathrm{T} 3$ & 620.7 & 78.6 & 102.7 & 82.3 & 51.3 & 935.7 & $315.0^{\mathrm{a}}$ \\
\hline
\end{tabular}

${ }^{1}$ T0 = Dieta control; T1: Dieta suplementada con 1.0\% de aceite de pescado; T2: Dieta

suplementada con $4.0 \%$ de semilla de sacha inchi; y T3: Dieta suplementada con $1.0 \%$ de aceite de pescado y $4.0 \%$ de semilla de sacha inchi

${ }^{a}$ Letras iguales dentro de columnas indican que no hay diferencia esta dística

Cuadro 2. Consumo semanal de alimento en materia seca (g) en cuyes con dietas suplementadas con aceite de pescado y sacha inchi (Plukenetia volubilis)

\begin{tabular}{cccccc}
\hline \multirow{2}{*}{ Tratamiento $^{1}$} & \multicolumn{4}{c}{ Semanas de suplemento } & \multirow{2}{*}{$\begin{array}{c}\text { Consumo } \\
\text { total }\end{array}$} \\
\cline { 2 - 5 } & 1 & 2 & 3 & 4 & $1207.9^{\mathrm{a}}$ \\
\hline T0 & 231.8 & 279.5 & 302.8 & 393.8 & $1209.6^{\mathrm{a}}$ \\
T1 & 234.2 & 283.3 & 316.1 & 376.0 & $1223.5^{\mathrm{a}}$ \\
T2 & 234.8 & 287.9 & 319.9 & 380.9 & $1171.6^{\mathrm{a}}$ \\
T3 & 235.4 & 281.8 & 305.7 & 348.7 & \\
\hline
\end{tabular}

${ }^{1}$ T0 = Dieta control; T1: Dieta suplementada con 1.0\% de aceite de pescado; T2: Dieta

suplementada con $4.0 \%$ de semilla de sacha inchi; y T3: Dieta suplementada con $1.0 \%$ de aceite de pescado y $4.0 \%$ de semilla de sacha inchi

${ }^{a}$ Letras iguales dentro de columnas indican que no hay diferencia esta dística

- Análisis químico proximal del alimento. El análisis de las cuatro dietas experimentales y del forraje fue realizado en el Laboratorio de Evaluación Nutricional de Alimentos (LENA) del Departamento Académico de Nutrición de la Facul- tad de Zootecnia, UNALM. Se pesaron $1000 \mathrm{~g}$ de cada dieta por tratamiento, se depositaron en bolsas plásticas y se remitieron al laboratorio. El mismo procedimiento se hizo con el forraje. 
Cuadro 3. Conversión alimenticia en cuyes con dietas suplementadas con aceite de pescado y sacha inchi (Plukenetia volubilis)

\begin{tabular}{|c|c|c|c|c|c|}
\hline \multirow{2}{*}{ Tratamiento ${ }^{1}$} & \multicolumn{4}{|c|}{ Semanas de suplemento } & \multirow{2}{*}{ Promedio } \\
\hline & 1 & 2 & 3 & 4 & \\
\hline $\mathrm{T} 0$ & 4.0 & 3.0 & 3.7 & 4.4 & $3.73^{\mathrm{a}}$ \\
\hline $\mathrm{T} 1$ & 3.5 & 2.9 & 3.6 & 4.2 & $3.53^{\mathrm{a}}$ \\
\hline $\mathrm{T} 2$ & 3.3 & 3.1 & 3.5 & 3.9 & $3.47^{\mathrm{a}}$ \\
\hline $\mathrm{T} 3$ & 3.0 & 2.7 & 3.7 & 4.8 & $3.55^{\mathrm{a}}$ \\
\hline
\end{tabular}

\section{Resultados Y Discusión}

\section{Ganancia de Peso}

El peso y la ganancia de peso por tratamiento se muestran en el Cuadro 1. Los cuyes de la dieta $\mathrm{T} 2$ con semilla de sacha inchi lograron la mayor ganancia de peso $(353.0 \mathrm{~g})$ y mayor peso $(983 \mathrm{~g})$ a las 4 semanas de iniciado el tratamiento, siguiendo en orden decreciente los cuyes de los tratamientos $\mathrm{T} 1$, T3 y T0, aunque estas diferencias no llegaron a ser estadísticamente significativas. El peso obtenido se encuentra dentro del peso comercial de cuyes $(>850 \mathrm{~g}$ ) (Morales et al., 2011) y comparable con lo obtenido en granjas comerciales (J. Guevara, Lima, comunicación personal). Estos resultados indican que el uso de dietas mejoradas, con aceite de pescado o con semillas de sacha inchi, no presentó ventajas en términos de rendimiento productivo en comparación con el uso de dietas no mejoradas (T0).

En otros estudios, Avdalov et al. (1992) mencionan que se obtienen mejores ganancias de peso en cerdos que consumen dietas que aportan ácidos grasos esenciales. Asi- mismo, Mattos et al. (2003) obtuvieron mejores ganancia de peso usando mayores porcentajes de ensilado de pescado en la ración.

\section{Consumo de Alimento}

El consumo de alimento en términos de materia seca fue similar entre los cuatro grupos experimentales y sin diferencia significativa (Cuadro 2). Por otro lado, en el estudio realizado por Mattos et al. (2003), encuentran un consumo superior en cuyes con dietas conteniendo mayores porcentajes de ensilado de pescado, probablemente debido a los ácidos grasos poliinsaturados.

\section{Conversión Alimenticia}

La conversión alimenticia semanal siguió una tendencia similar a la ganancia de peso y al consumo de alimento; es decir, fue ligeramente superior en los cuyes que recibieron la dieta con semilla de sacha inchi, seguido de los cuyes alimentados con la dieta de aceite de pescado, los cuyes de la dieta con ambos ingredientes y la menor conversión se observó en los cuyes de dieta control. Sin embargo, las diferencias observadas no fueron estadísticamente diferentes (Cuadro 3). 
Estos valores difieren a los publicados por Cerna (1997), quién encontró valores de conversión alimenticia de 3.03 a 3.26, superiores a las conversiones promedio de las cuatro semanas $(3.47$ - 3.73) del presente trabajo. No obstante, los resultados son coincidentes con las conversiones alimenticias obtenidas por Dulanto (1999). Por otro lado, se ha reportado una tendencia en la mejora de la conversión alimenticia en dietas a base de ensilado de pescado, debido a que su energía es aportada a través de los ácidos grasos que son metabólicamente más eficientes en ser utilizados (Ottati y Bello, 1992).

Esto permite concluir que las ganancias de peso y las conversiones alimenticias alcanzadas en este experimento son señales de un comportamiento productivo eficiente y comparable a lo que se logra en las granjas comerciales de cuyes, con la ventaja de que la carne de cuy contiene ácidos grasos omega-3 para beneficio de la salud de los consumidores (Guevara et al., 2009).

\section{Rendimiento de Carcasa}

El rendimiento de carcasa fue estadísticamente similar en los cuatro tratamientos (T0: 70.7\%; T1: 69.5\%; T2: 71.7\%; T3: 71.2\%). Estos hallazgos fueron similares a los obtenidos por Cerna (1997), quien obtuvo rendimientos de carcasa entre $70.9 \mathrm{y}$ $72.7 \%$.

\section{Literatura Citada}

1. Avdalov N, Barlocoo N, Bauza R, Bertullo E, Corengia C, Giacommeti L, Panucio A. 1992. Evaluación del ensilado biológico de pescado en la alimentación de cerdos en engorde. En: Segunda Consulta de Expertos sobre Tecnología de Productos Pesqueros en América Latina. Roma: FAO, Informe de Pesca 441: 88-98.
2. Camino J, Hidalgo V. 2014. Evaluación de dos genotipos de cuyes (Cavia porcellus) alimentados con concentrado y exclusión de forraje verde. Rev Inv Vet Perú 25: 190-197. doi: 10.15381/ rivep.v25i2.8490

3. Cerna A. 1997. Evaluación de cuatro niveles de residuo cervecería seco en el crecimiento y engorde de cuyes. Tesis de Ing. Zootecnista. Lima: Facultad de Zootecnia, Universidad Nacional Agraria La Molina. 63 p.

4. Dulanto M. 1999. Parámetros productivos y reproductivos de tres líneas puras y dos grados de cruzamiento entre líneas de cuyes. Tesis de Bachiller. Lima: Facultad de Zootecnia, Universidad Nacional Agraria la Molina. $65 \mathrm{p}$.

5. Follegatti L, Piantino C, Grimaldi R. Cabral F. 2009. Supercritical $\mathrm{CO}_{2}$ extraction of omega-3 rich oil from Sacha inchi (Plukenetia volubilis L.) seeds. J Supercrit Fluid 49: 323-329. doi 10.1016/ j.supflu.2009.03.010

6. Gorriti A, Arroyo JT, Quispe F, Cisneros B, Condorhuamán $M$, Almora Y, Chumpitaz V. 2010. Toxicidad oral a 60 días del aceite de sacha inchi (Plukenetia volubilis L.) y linaza (Linum usitatissimum L.) y determinación de la dosis letal 50 en roedores. Rev Peru Med Exp Salud Pública 27: 352360 . doi: $10.1590 / \mathrm{S} 1726-$ 46342010000300007

7. Gutiérrez L, Rosada L, Jiménez A. 2011. Chemical composition of sacha inchi (Plukenetia volubilis L.) seeds and characteristics of their lipid fraction. Grasas Aceites 62: 76-83. doi: 10.3989/ gya044510

8. Hosamani KM, Ganjihal S. 2003. Unique occurrence of unusual fatty acids in Ochrocarpus africanus seed oil. Ind Crop Prod 18: 111-116. doi: 10.1016/ S0926-6690(03)00031-1

9. Mattos J, Chauca L, San Martín F, Carcelén F, Arbaiza T. 2003. Uso del ensilado biológico de pescado en la ali- 
mentación de cuyes mejorados. Rev Inv Vet Perú 14: 89-96. doi: 10.15381/ rivep.v14i2.1612

10. Morales A, Carcelén F, Ara M, Arbaiza T, Chauca L. 2011. Evaluación de dos niveles de energía en el comportamiento productivo de cuyes (Cavia porcellus) de la raza Perú. Rev Inv Vet Perú 22: 177-182. doi: 10.15381/ rivep.v22i3.254

11. Ottati G, Bello R. 1992. Ensilado microbiano de pescado en la alimenta ción porcina. I. Valor nutritivo del producto en dietas para cerdos. En: Consulta de Expertos sobre Tecnología de Productos Pesqueros en América Latina. Roma: FAO, Informe de Pesca 441: 69-79.

12. Sánchez A, Sánchez S, Godoy S, Díaz $R$, Vega N. 2009. Gramíneas tropicales en el engorde de cuyes mejorados sexados (Cavia porcellus Linnaeus) en la zona de La Maná. Cienc Tecnol 2: 25-28. 